\title{
Article
}

\section{Electron density errors and density-driven exchange-correlation energy errors in approximate density functional calculations}

\author{
Pal Daniel Mezei, Gabor I. Csonka, and Mihaly Kallay
}

J. Chem. Theory Comput., Just Accepted Manuscript • DOI: 10.1021/acs.jctc.7b00550 • Publication Date (Web): 11 Sep 2017

Downloaded from http://pubs.acs.org on September 12, 2017

\section{Just Accepted}

"Just Accepted" manuscripts have been peer-reviewed and accepted for publication. They are posted online prior to technical editing, formatting for publication and author proofing. The American Chemical Society provides "Just Accepted" as a free service to the research community to expedite the dissemination of scientific material as soon as possible after acceptance. "Just Accepted" manuscripts appear in full in PDF format accompanied by an HTML abstract. "Just Accepted" manuscripts have been fully peer reviewed, but should not be considered the official version of record. They are accessible to all readers and citable by the Digital Object Identifier (DOI®). "Just Accepted" is an optional service offered to authors. Therefore, the "Just Accepted" Web site may not include all articles that will be published in the journal. After a manuscript is technically edited and formatted, it will be removed from the "Just Accepted" Web site and published as an ASAP article. Note that technical editing may introduce minor changes to the manuscript text and/or graphics which could affect content, and all legal disclaimers and ethical guidelines that apply to the journal pertain. ACS cannot be held responsible for errors or consequences arising from the use of information contained in these "Just Accepted" manuscripts. 


\title{
Electron density errors and density-driven exchange-correlation energy errors in approximate density functional calculations
}

\author{
Pál D. Mezei, ${ }^{1 *}$ Gábor I. Csonka ${ }^{2}$, and Mihály Kállay ${ }^{1}$ \\ ${ }^{1}$ MTA-BME Lendület Quantum Chemistry Research Group, Department of Physical Chemistry and \\ Materials Science, Budapest University of Technology and Economics, H-1521 Budapest, Hungary \\ ${ }^{2}$ Department of Inorganic and Analytical Chemistry, Budapest University of Technology and \\ Economics, H-1521 Budapest, Hungary
}

*E-mail: mezei.pal@mail.bme.hu

\begin{abstract}
Since its formal introduction, density functional theory has achieved many successes on the fields of molecular and solid-state chemistry. According to its central theorems, the ground state of a manyelectron system is fully described by its electron density, and the exact functional minimizes the energy at the exact electron density. For many years of density functional development, it was assumed that the improvements in the energy are accompanied by the improvements in the density, and the approximations approach the exact functional. In a recent analysis (Medvedev et al. Science 2017, 355, 49-52.), it has been pointed out for fourteen first row (Be-Ne) atoms and cations with 2, 4, or 10 electrons that the nowadays popular flexible but physically less rigorous approximate density functionals may provide large errors in the calculated electron densities despite the accurate energies. Although far-reaching conclusions have been drawn in this work, the methodology used by the authors may need improvements. Most importantly, their benchmark set was biased towards small atomic cations with compressed, high electron densities. In our paper, we construct a molecular test set with chemically relevant densities and analyze the performance of several density functional approximations including the less-investigated double hybrids. We apply an intensive error measure for the density, its gradient, and its Laplacian and examine how the errors in the density propagate into the semi-local exchange-correlation energy. While we have confirmed the broad conclusions of Medvedev et al., our different way of analyzing the data has led to conclusions that differ in detail. Finally, seeking for a rationale behind the global hybrid or double hybrid methods from the density's point of view, we also analyze the role of the exact exchange and second-order perturbative correlation mixing in PBE-based global hybrid and double hybrid functional forms.
\end{abstract}

\section{Introduction}

Density functional theory is an essential tool in the electronic structure theory of molecules and materials, which provides accurate results at a low computational cost. ${ }^{1,2}$ According to the HohenbergKohn theorems, ${ }^{3}$ the ground-state electron density uniquely determines the potential and thus contains all information about the system. There exists a universal density functional, which yields the exact ground-state energy of the system from its exact electron density variationally. In principle, the exact solution is possible using the simple one-electron Kohn-Sham equations ${ }^{4}$ since the potential of the physical interacting system can be replaced by the potential of a fictitious non-interacting system. The main difficulty is that a general form for the universal functional is not known. Several approximate functionals were constructed taking into account known physical properties of the exact exchangecorrelation functional, ${ }^{5,6,7}$ but many were empirically fitted for databases with various chemical properties. ${ }^{8,9,10}$ The exchange and correlation parts are usually approximated together for systematic error 
cancellation. ${ }^{7}$ During the development, it was assumed that functionals with better energetics reproduce better the exact Kohn-Sham potential and thus produce better density. ${ }^{11,12,13}$ However, density functional approximations are close to the exact functional if they estimate well the exact Kohn-Sham potential.

There are several studies in the literature assessing the quality of the electron density and its derivatives obtained with various density functional approximations. For small molecules, Boyd et al. compared the electron density and its Laplacian at the bond critical point obtained from density functional and wave function calculations and found that three-parameter hybrid density functionals ${ }^{14}$ perform better than their semi-local parent functionals and much better than the local density approximation (LDA). ${ }^{15}$ Csonka et al. ${ }^{16}$ showed for the hydrogen molecule that the electron density can be accurately reproduced along the bond axis around the bond critical point by a proper mixing of the exact and semilocal exchange in a B3P86-type functional. ${ }^{17,18}$ The corresponding exact Kohn-Sham potential $\left(v_{K S}\right)$ was also presented, which depends on the density $(n)$, its gradient $(\nabla n)$, its Laplacian $\left(\nabla^{2} n\right)$, and the energy of the (highest) occupied orbital $(\varepsilon) .{ }^{19}$ (Note that this form is universal for two-electron spin-singlet systems.)

$v_{K S}(\boldsymbol{r})=-\frac{1}{8} \frac{(\nabla n)^{2}}{n^{2}}+\frac{1}{4} \frac{\nabla^{2} n}{n}+\varepsilon$

Furthermore, the errors of approximate Kohn-Sham potentials were analyzed in the bond critical point of the dissociating hydrogen molecule, where the exact Kohn-Sham potential is determined by the density, its Laplacian, and the energy of the (highest) occupied orbital since the gradient is zero. ${ }^{16}$ Bochevarov and Friesner compared the electron densities of small molecules obtained from selfconsistent density functional calculations to full configuration interaction level reference densities. ${ }^{20}$ They concluded that certain generalized gradient approximation (GGA) or three-parameter hybrid $\mathrm{GGA}^{14}$ functionals perform better with the empirically fitted OPTX exchange ${ }^{21}$ than with the original B88 exchange. ${ }^{17}$ Grabowski, Teale, et al. observed for standard local and semi-local functionals with the $\mathrm{VWN}^{22}$ and $\mathrm{LYP}^{23}$ correlation that the correlated densities (i.e., the difference between the densities generated by the exchange-correlation method and the corresponding exchange-only method) and the correlation potentials are qualitatively incorrect, although the correlation may provide some error cancellation with the exchange part. ${ }^{24}$ Grabowski, Fabiano, et al. also suggested the integral absolute density differences to measure the approximate density functional density errors radially in spherical atoms and along the bond axis in diatomic molecules. ${ }^{25}$ According to the analysis of Tognetti and Joubert on 50 small molecules, ${ }^{26}$ the electron density and its Laplacian at the bond critical point can be better described by the meta-GGA level TPSS functional ${ }^{27}$ than by the GGA-level PBE functional. ${ }^{28}$ However, the accuracy of the former can be achieved at the GGA-level by the empirically fitted HCTH functional, which was optimized also for some accurate exchange-correlation potentials. ${ }^{29,30,31}$ In contrast to the above mentioned studies, Cruz et al. showed that functionals which yield accurate energies often produce wrong potentials. ${ }^{32}$ Finally, Kim et al. argued that there are situations (e.g., electron affinities) when the density-driven error (i.e., the difference of the total energies evaluated on the approximate and the exact density) dominates over the functional error in the error of a variational density functional calculation. ${ }^{33}$

Very recently, Medvedev et al. have reported that the unconstrained and highly empirical density functional construction with flexible functional forms can also bring farther from the exact functional as such functionals give accurate energies on wrong densities. ${ }^{34}$ Their flexible functional form can be easily overfitted, which leads to oscillations in the exchange-correlation enhancement factor. These oscillations can propagate into the exchange-correlation potential and thus to the density.

Although this report met an intense response in the literature, ${ }^{35,36}$ it has been criticized how the authors arrived at their conclusion. ${ }^{37,38}$ In a comment, ${ }^{37}$ Kepp pointed out that the benchmark set was strongly biased towards small atoms and atomic cations with $1 \mathrm{~s}^{2}$ or $1 \mathrm{~s}^{2} 2 \mathrm{~s}^{2}$ electronic structure, and it is unclear if the description of the very compact electron densities in the atomic cations (with large dynamic 
correlation) is chemically relevant. The test set seems to favor the hybrid functionals $\left(e . g\right.$., $\left.\mathrm{PBE} 0^{39}\right)$ since the Hartree-Fock (HF) exchange gives more accurate densities for two-electron systems. In a systemwise comparison, Kepp found a strong linear relationship between the errors in the ionic densities and the errors in the ionic energy differences computed by semi-local or hybrid functionals. In their response, ${ }^{40}$ Medvedev et al. emphasized that the atoms and atomic cations were only intended to reveal internal problems in the methods, the ranking of the HF method is only mediocre because of its maximum error, and the relationship of the ionic densities and energy differences is evident as the density and energy errors each scales with the nuclear charge at constant electron number. Some other issues were collected by Korth. ${ }^{38}$ For example, the all-electron coupled-cluster singles and doubles (CCSD) reference method in this benchmark might miss some core-valence correlation for systems with more than two electrons due to the missing triple or higher-order excitations, although higher level densities probably do not change the qualitative picture. ${ }^{38}$ Also note that the quantity representing the functional errors is extensive and not practical to compare molecular densities.

Brorsen et al. presented a correspondence between the errors in the computed diatomic molecular densities near the bond critical point, and the errors in the computed molecular atomization energies for several semi-local and hybrid functionals (as well as one double hybrid). ${ }^{41}$ For some recent highly empirical hybrid functionals, the errors in densities and atomization energies were decoupled. Gould argued that the atomic test set of Medvedev et al. ${ }^{34}$ is biased towards the high density limit, and their analysis based also on the gradients and Laplacians is biased towards the core regions. ${ }^{42}$ In addition to the densities, he also proposed testing the calculated left Fukui functions on a test set constituted of Li, $\mathrm{C}$, and $\mathrm{F}$ atoms and found that a couple of functionals (i.e., SOGGA11X, ${ }^{43} \mathrm{TPSSh}^{44}$ and B2PLYP ${ }^{45}$ ) can be good both at densities and Fukui functions. Verma and Truhlar investigated the dipole moments of organic and inorganic compounds and found that density functionals describe better the charge distribution of single-reference systems than multi-reference systems. ${ }^{46}$ In their analysis, the best performers were global hybrid functionals (i.e., B97-1, ${ }^{29}$ PBE0, ${ }^{39}$ TPSSh, $^{44}$ and PW6B95 ${ }^{47}$ ) with relatively small fraction of exact exchange and a screened hybrid functional (i.e., HSE06 ${ }^{48}$ ).

In this paper, we analyze several functionals from various rungs of the Jacob's ladder ${ }^{49}$ of density functional approximations according to their density errors and density-driven exchange-correlation energy errors. In our modified methodology, we use composite coupled-cluster singles, doubles, triples, and quadruples (CCSDTQ) reference densities, and an intensive error measure. This way, our conclusions will be somewhat different even for the atomic density test set $\left(\mathrm{B}^{3+}, \mathrm{C}^{4+}, \mathrm{N}^{5+}, \mathrm{O}^{6+}, \mathrm{F}^{7+}, \mathrm{Ne}^{8+}\right.$, $\mathrm{Be}, \mathrm{B}^{+}, \mathrm{C}^{2+}, \mathrm{N}^{3+}, \mathrm{O}^{4+}, \mathrm{F}^{5+}, \mathrm{Ne}^{6+}, \mathrm{Ne}$ ) of Medvedev et al. ${ }^{34}$ Furthermore, we construct another test set from small molecules $\left(\mathrm{H}_{2}, \mathrm{LiH}, \mathrm{Li}_{2}, \mathrm{LiF}, \mathrm{BH}_{3}, \mathrm{H}_{2} \mathrm{O}, \mathrm{HF}, \mathrm{CO}, \mathrm{N}_{2}, \mathrm{~F}_{2}\right)$ with densities more relevant in chemistry. Additionally, we also discuss if there is a rationale behind the global hybrid or double hybrid functionals from the density's point of view. Since many parametrizations have been suggested in the literature for the PBE hybrid (PBEh) ${ }^{39,50,51}$ or PBE double hybrid (PBE-DH) ${ }^{52,53,54}$ functionals, we have studied the ratio of exact exchange and/or the ratio of second-order perturbative (PT2) correlation in these wellinvestigated forms.

\section{Methodology and computational details}

All the computations were performed with the MRCC quantum chemistry software. ${ }^{55}$ To avoid any ambiguity, a close to infinite integration grid was used in the calculations: a 420-point Log3 radial grid (or a 415-point Log3 grid for hydrogen atoms), ${ }^{56}$ and a 1202-point angular Lebedev grid. ${ }^{57}$ For the calculations, the aug-cc-pVXZ $Z^{58}$ and aug-cc-pwCVXZ $Z^{59}(X=\mathrm{D}, \mathrm{T}, \mathrm{Q}, 5)$ basis set families were applied. For the atomic density test set $\left(\mathrm{B}^{3+}, \mathrm{C}^{4+}, \mathrm{N}^{5+}, \mathrm{O}^{6+}, \mathrm{F}^{7+}, \mathrm{Ne}^{8+}, \mathrm{Be}, \mathrm{B}^{+}, \mathrm{C}^{2+}, \mathrm{N}^{3+}, \mathrm{O}^{4+}, \mathrm{F}^{5+}, \mathrm{Ne}^{6+}, \mathrm{Ne}\right)$ of Medvedev et al., ${ }^{34}$ we computed all-electron CCSD/aug-cc-pwCV5Z for the two-electron systems and 
composite all-electron CCSDTQ/aug-cc-pwCV5Z densities otherwise. The latter is defined by eqs 2 and 3 , where $n_{\text {basis }}^{\text {method }}$ is the density evaluated with a particular method and basis set.

$n_{\text {aug-cC-pwCV5Z }}^{\text {Comp.CCSDT }}=n_{\text {aug-cc-pwCV5Z }}^{C C S D}+n_{\text {aug-cc-pwCVQZ }}^{C C S D T}-n_{\text {aug }-c c-p w C V Q Z}^{C C S D}$

$n_{\text {aug-cC-pwCV5Z }}^{\text {comp.CCSDTQ }}=n_{\text {aug-cc-pwCV5Z }}^{\text {comp.CCST }}+n_{\text {aug-cc-pwCVTZ }}^{C C S D T Q}-n_{\text {aug }-c c-p w C V T Z}^{C C S D T}$

For our new molecular density test set $\left(\mathrm{H}_{2}, \mathrm{LiH}, \mathrm{Li}_{2}, \mathrm{LiF}, \mathrm{BH}_{3}, \mathrm{H}_{2} \mathrm{O}, \mathrm{HF}, \mathrm{CO}, \mathrm{N}_{2}, \mathrm{~F}_{2}\right)$, we computed CCSD/aug-cc-pV5Z density for the $\mathrm{H}_{2}$ molecule and composite all-electron CCSDTQ/aug-cc-pwCV5Z densities [defined by eqs 2 and 3, but the effect of the quadruple excitations was considered with the augcc-pwCVTZ(-f) basis set] for the larger molecules. In our calculations, we used experimental molecular geometries taken from the NIST Computational Chemistry Comparison and Benchmark Database. ${ }^{60}$

To represent the important rungs (semi-local and non-local) of the Jacob's ladder of density functional approximations, we selected five GGA (i.e., SOGGA11, ${ }^{61} \mathrm{BLYP},{ }^{17,23} \mathrm{BP} 86,{ }^{17,18} \mathrm{PBE},{ }^{28}$ RGE2 ${ }^{62}$ ), five meta-GGA (i.e., PKZB $,{ }^{63} \mathrm{M} 06 \mathrm{~L},{ }^{64} \mathrm{SCAN},{ }^{65} \mathrm{MS} 0,{ }^{66} \mathrm{TPSS}^{27}$ ), five hybrid (i.e., M062X, ${ }^{67}$ SOGGA11X, ${ }^{43}$ B3LYP,${ }^{14}$ PBE0, ${ }^{39}$ TPSSh $^{44}$ ), and five double hybrid (i.e., B2PLYP ${ }^{45}$ B2GPPLYP, ${ }^{68}$ PBE0-DH, $\left.{ }^{52} \mathrm{PBE}-\mathrm{QIDH},{ }^{53} \mathrm{PBE} 0-2^{54}\right)$ functionals with either physical or empirical construction. Additionally, we mapped the one- $\left(a_{\mathrm{X}}\right)$ and two-dimensional $\left(a_{\mathrm{X}}, a_{\mathrm{C}}\right)$ parameter space in the PBEh (eq 4) and PBE-DH (eq 5) forms:

$E_{X C}^{P B E h}[n]=a_{X} E_{X}^{\text {exact }}[n]+\left(1-a_{X}\right) E_{X}^{P B E}[n]+E_{C}^{P B E}[n]$

$E_{X C}^{P B E-D H}[n]=a_{X} E_{X}^{\text {exact }}[n]+\left(1-a_{X}\right) E_{X}^{P B E}[n]+a_{C} E_{C}^{P T 2}[n]+\left(1-a_{C}\right) E_{C}^{P B E}[n]$,

where $E_{X}^{\text {exact }}$ is the exact exchange energy, $E_{X}^{P B E}$ and $E_{C}^{P B E}$ denote the PBE exchange and correlation energies, respectively, and $E_{C}^{P T 2}$ stands for the PT2 correlation energy.

To assess the performance of the various density functional approximations, we propose here a normed integral absolute deviation (NIAD) of the density functional (DF) and coupled-cluster (CC) densities and density derivatives ( $p=n$ for the density, $p=|\nabla n|$ for its gradient, and $p=\nabla^{2} n$ for its Laplacian) similar to the integral absolute deviation (IAD) applied earlier by Grabowski, Fabiano, et al. radially in spherical atoms and along the bond axis in diatomic molecules: ${ }^{25}$

$N I A D[p]=\frac{1}{N} \int\left|p_{D F}(\boldsymbol{r})-p_{C C}(\boldsymbol{r})\right| d^{3} \boldsymbol{r} \stackrel{\text { spherical }}{\longrightarrow} \frac{1}{N} \int\left|4 \pi r^{2} p_{D F}(r)-4 \pi r^{2} p_{C C}(r)\right| d r$

where $N$ is the number of electrons. This error measure is uniformly-distributed over all the electrons in space, which can be clearly demonstrated for spherically symmetric atoms, where the integration is over the radial density errors (for examples, see Figures S1-3 in the supporting information). In other words, the radial density near the nucleus is weighted equally to the radial density far from the nucleus. Another advantage of this error measure is that it can also be applied to non-spherical molecular systems. We chose the density errors per electron because we can directly compare this way the density errors for different atoms as opposed to the density errors per atom, which depends on the number of electrons in the atom. We retain this choice also for the molecules because we can compare the atomic and molecular density errors to each other, in contrast to the density errors per bond, which cannot be applied for atoms. In contrast to the suggestion of Gould ${ }^{42}$ that the inclusion of the gradient and Laplacian would bias the analysis towards the core regions, we also consider these quantities since the importance of the gradient and Laplacian can be supported by the universal formula for the exact Kohn-Sham potential of the twoelectron spin-singlet systems (see eq 1). Moreover, the Laplacian may be particularly important in molecules as the Laplacian appears even at the bond critical point in the potential of the two-electron spin-singlet molecules like $\mathrm{H}_{2}$.

In thermochemical benchmarks, the calculated energies are usually said to be accurate if the errors in energy differences are lower than some threshold, for example, $1 \mathrm{kcal} \mathrm{mol}^{-1}$. The choice of such absolute threshold is, however, not so convenient to assess whether the calculated densities are accurate or not. For this reason, we need to set up a relative scale for the magnitude of the errors. A natural choice 
is to compare the errors in the semi-local or non-local density functional densities to the errors in the LDA (namely the SVWN5) ${ }^{69,22}$ densities. Note that the "average functional" as suggested by Medvedev et al. ${ }^{34}$ depends on the functionals considered in the benchmark. Another natural choice would be the Hartree method with completely missing exchange and correlation. A further choice would be the HF method, but it is not a particularly suitable reference for density functional methods due to the fully exact treatment of the exchange and the completely missing electron correlation. Our test calculations show that comparing the errors to the LDA and Hartree density errors leads to the same ranking of the methods (see the Supporting Information).

For each descriptor $p$, we define the relative average normed error (RANE) as a dimensionless error measure.

$R A N E[p]=\frac{\sum_{\text {system }} N I A D_{\text {system }}^{\text {method }}[p] / \mathcal{N}_{\text {sys }}}{\sum_{\text {system }} N I A D_{\text {system }}^{L D A}[p] / \mathcal{N}_{\text {sys }}}$,

where the sum runs over the systems of the test set, NIAD system is the NIAD defined by eq 6 calculated for the corresponding system with the given method, and $\mathcal{N}_{s y s}$ is the number of systems in the test set. The overall RANE is the average of the RANEs for the three descriptors.

In addition to the density errors, we show here the density-driven exchange-correlation energy errors per particle in the PBE exchange-correlation energy evaluated on the various approximate densities (for the sake of simplicity, we abbreviate this quantity as DDXC):

$D D X C=\frac{E_{X C}^{P B E}\left[n_{D F}\right]-E_{X C}^{P B E}\left[n_{C C}\right]}{N}$,

where $n_{D F}$ and $n_{C C}$ stand for the densities evaluated with the DF approximation and the reference CC method, respectively. Please do not confuse this quantity with the density-driven error defined by Kim et al. for total energies. ${ }^{33}$ For a given test set, we show here the mean, mean absolute, and standard deviation DDXC. We assume that the DDXC does not depend much on the choice of the semi-local density functional approximation since the density-driven errors agree well in the non-empirical PBE and BLYP exchange-correlation energies (see Figure S4). These density-driven exchange-correlation energy errors also correlate with the ones in the semi-empirical SOGGA11 exchange-correlation energies, albeit the density-driven error in the SOGGA11 exchange-correlation energy significantly differs from the density-driven errors in the PBE or BLYP exchange-correlation energies on the SOGGA11 densities. This may indicate the opportunity for some internal error cancellation in this empirical method, but it does not follow directly from our results since we did not study here either the functional error (i.e., the difference between the total energy obtained by evaluating the approximate functional at the exact electron density and exact total energy) or the density-driven total energy error.

\section{Results and discussion}

Our methodology in the analysis of the atomic $\left(\mathrm{B}^{3+}, \mathrm{C}^{4+}, \mathrm{N}^{5+}, \mathrm{O}^{6+}, \mathrm{F}^{7+}, \mathrm{Ne}^{8+}, \mathrm{Be}, \mathrm{B}^{+}, \mathrm{C}^{2+}, \mathrm{N}^{3+}\right.$, $\mathrm{O}^{4+}, \mathrm{F}^{5+}, \mathrm{Ne}^{6+}, \mathrm{Ne}$ ) density errors leads to somewhat different conclusions than those obtained by Medvedev et al. ${ }^{34}$ In their analysis, the empirical M06L meta-GGA functional seemed to produce atomic densities similar to the LDA method. The PBE0 global hybrid GGA functional showed better performance on atomic densities than the TPSS meta-GGA and TPSSh global hybrid meta-GGA functionals. Contrarily, our comparison (Figure 1a) shows that the M06L functional produces clearly better atomic densities than the LDA method. The TPSS and TPSSh methods provide clearly better atomic densities than the PBE0 method. The observed differences can originate from several sources. (i) As an error measure, we apply here the normed (not size-extensive) integral absolute deviations instead of the size-extensive root mean square deviations on a radial grid. (ii) Our comparison relies on the average errors instead of the maximal errors (or on their combination). (iii) We normalize the errors to 
the LDA errors instead of the "average functional" errors. (iv) Our composite CCSDTQ reference densities are more accurate for the many-electron systems than the CCSD reference densities.

The average CCSD errors are small for the atomic test set (see Figure 1a), and thus using the composite CCSDTQ densities as reference instead of the CCSD densities ${ }^{34}$ does not lead to qualitative changes (see also Figure S5). Normalization of the semi-local and non-local density functional errors to the LDA errors does not significantly change the picture either (see Figure S5) compared to the normalization to the "average functional" errors ${ }^{34}$ because the improvements in the semi-local or nonlocal density functional errors upon the LDA errors seem to be more or less simultaneous for the three descriptors (see also Figure 1a). According to our analysis, the RANEs and the size extensive relative average errors (RAEs), both relative to LDA, correlate relatively well (see Figure S5), although this can be a minor source of the observed differences. For example, the different ranking of the TPSS and PBE0 methods comes from the application of an intensive error measure instead of an extensive one ( $c f$. the NIADs of these methods in Table S1). The overall relative maximum error (RME) is 1.34 for the TPSS method, which originates from the density and the Ne system, as well as 1.12 for the PBE0 method, which originates from the Laplacian and the $\mathrm{Ne}^{6+}$ system. While the overall relative maximum normalized error (RMNE) is 0.95 for the TPSS method, which originates from the density and the Be system, as well as 0.98 for the PBE0 method, which originates from the Laplacian and the $\mathrm{Ne}^{8+}$ system. Considering the RANEs instead of the relative maximum normed errors (RMNEs), highly influences the relative order of the methods (see Figure S5). Thus, using average errors instead of maximum ones seems to be the major source of the observed differences. Notice that ranking the methods according to their maximum errors would put too much weight on the Laplacian and on the highly-charged systems in the comparison (see Figure S6). Considering either the RME or RMNE error measures, 9 methods out of 25 are judged by their Laplacian errors. Moreover, considering the RME error measure, 7 methods out of 25 are judged by the Laplacian errors on the $\mathrm{Ne}^{6+}$ system. While considering the RMNE error measure, 8 methods out of 25 are judged by the Laplacian errors on the $\mathrm{Ne}^{8+}$ system. On the other side, ranking the methods according to their overall RANE errors puts equal weight on the density, gradient, and Laplacian, as well as on the individual systems, even though a large part of the atomic test set (6 out of 14) includes two-electron systems.

Since most of the semi-local and non-local density functionals simultaneously improve the atomic densities, gradients, and Laplacians, the inclusion of the Laplacian in our comparison does not distort our analysis much. The errors for the empirical SOGGA11, M06L, SOGGA11X, and M062X functionals are higher in the Laplacians than in the densities or gradients; however, the overall errors are only moderately larger than the errors in the densities and gradients. Contrarily, the RGE2 method gives somewhat larger errors for the densities and smaller errors for the gradients and Laplacians than the similar PBE method. Moreover, the HF method shows an uneven performance with quite erroneous densities but with good gradients and Laplacians, although its overall performance for atomic densities and density derivatives is similar to the best meta-GGA or hybrid functionals (i.e., TPSS and TPSSh). Interestingly, more exact exchange leads to more accurate density Laplacians ( $c f$. BLYP, PBE, and TPSS with their global hybrid versions: B3LYP, PBE0, and TPSSh), even though the exact exchange mixing does not necessarily improve the densities if the semi-local part is reparametrized in the hybrid version ( $c f$. M06L and M062X). The correlation influences equally all the three density descriptors ( $c f$. BLYP and BP86). The B2GPPLYP double hybrid with $65 \%$ of exact exchange and $36 \%$ of PT2 correlation provides better atomic densities than the B2PLYP double hybrid with 53\% of exact exchange and $27 \%$ of PT2 correlation. Similarly, the accuracy of the PBE-based double hybrid atomic densities increases with the amount of exact exchange and PT2 correlation (PBE0-DH with 50\% of exact exchange and $12.5 \%$ of PT2 correlation < PBE-QIDH with $\sim 69 \%$ of exact exchange and $\sim 33 \%$ of PT2 correlation < PBE0-2 with $\sim 79 \%$ of exact exchange and $50 \%$ of PT2 correlation). The PBE0-DH functional performs 
similar to the B2PLYP functional with similar exact exchange and PT2 correlation ratios. The PBEQIDH functional is somewhat better for atomic densities than the B2GPPLYP functional with similar exact exchange and PT2 correlation ratios. Among the examined double hybrids, the PBE0-2 shows the best accuracy. The Møller-Plesset second-order perturbation theory (MP2) method gives slightly better atomic densities, but it performs worse than the CCSD method on the very compact cations because of the poor description of the relatively large dynamic correlation in these systems. Concerning the densities obtained with higher-order CC methods, the triple excitations have only a very small effect, while the quadruple excitations have practically no contribution.

For the molecular densities $\left(\mathrm{H}_{2}, \mathrm{LiH}, \mathrm{Li}_{2}, \mathrm{LiF}, \mathrm{BH}_{3}, \mathrm{H}_{2} \mathrm{O}, \mathrm{HF}, \mathrm{CO}, \mathrm{N}_{2}, \mathrm{~F}_{2}\right)$, the average NIAD density errors are somewhat larger than the corresponding average NIAD errors for atoms but the average NIAD gradient and Laplacian errors are significantly smaller (see Tables S1 and S2). The overall (threedescriptor) improvement in the NIAD errors is larger for the semi-local functionals than for the LDA method. However, the relative order of the density functional methods only slightly changes (Figure 1b). The SCAN meta-GGA functional performs better than the MS0 and TPSS functionals. The SOGGA11X global hybrid functional provides better molecular densities than the B3LYP method. The PBE0-DH global double hybrid functional is better than the B2PLYP method. The correlation becomes more important in the many-electron molecules. This can be followed as the HF method tends to give worse molecular densities, although its overall density errors are still comparable to the ones obtained with the best GGA methods. The best double hybrid method (PBE0-2) yields similar molecular densities to the MP2 method. The CCSD method works only slightly better for molecular densities. The CCSDT densities are almost identical to CCSDTQ densities again. In contrast to the atomic density test set, there is a stronger correlation between the average and maximum density errors, which shows that our molecular density test set is well-balanced.

For the different functionals, the density-driven error in the PBE (or other semi-local) exchangecorrelation energy does not always follow the same order we have previously observed for the density errors (Figure 2a). One possible explanation is that the locally positive or negative errors in the exchangecorrelation energy per particle cancel each other in the exchange-correlation energy integral. Also notice that the PBE exchange-correlation energy does not depend on the density Laplacian. For example, the density-driven error in the PBE exchange-correlation energy for the atoms is significantly smaller on the BP86 densities than on the RGE2 densities, where the overall density error is smaller only because of the Laplacian (see Figure 1a). Similarly, the density-driven error in the PBE exchange-correlation energy is smaller on the M06L densities than on the SCAN densities. The density-driven exchange-correlation energy error is more systematic on the densities calculated with the best semi-local or non-local methods than on the HF densities. As well as the MP2 atomic densities look only slightly better concerning the density-driven exchange-correlation energy error than the best hybrid or double hybrid atomic densities. (The correspondence between the atomic density errors and density-driven exchange-correlation energy errors can be followed in Figure S7.)

For the molecules, the density-driven errors in the PBE exchange-correlation energy (Figure 2b) are generally smaller than the errors we have observed for atoms. The meta-GGA densities lead to smaller density-driven exchange-correlation energy errors than the GGA densities ( $c f$. PBE with TPSS). This difference also remains among the hybrid functionals, where the density-driven exchange-correlation energy error is smaller on the global hybrid meta-GGA densities than on the global hybrid GGA densities ( $c f$. PBE0 with TPSSh). The molecular densities obtained by the best semi-local or non-local density functionals are better again than the molecular HF densities according to their density-driven exchangecorrelation energy errors. Furthermore, the density-driven error in the PBE exchange-correlation energy is slightly smaller on the PBE0-2 densities than on the MP2 densities. Considering both the molecular density errors and density-driven errors in the semi-local exchange-correlation energies (see Figure S8), 
the best performers among their kind in the order of increasing accuracy are the PBE GGA functional, the TPSS and SCAN meta-GGA functionals, the TPSSh hybrid functional, and the PBE0-2 double hybrid functional. Notice that all these methods have non-empirical origin, which supports the main conclusion of Medvedev et al. ${ }^{34}$

Seeking for an optimal exact exchange mixing in the PBE hybrid form, we changed (from $0 \%$ to $100 \%$ by $5 \%$ ) the ratio of exact exchange. On the atomic densities, the optimal exact exchange mixing ratios were found at $\sim 40 \%$ for the density, at $\sim 80 \%$ for the gradient, at $\sim 85 \%$ for the Laplacian, and at $\sim 80 \%$ for the three descriptors since the error in the Laplacian of the density is very sensitive to the ratio of exact exchange (Figure 3a). However, such high fraction of exact exchange only seems to be optimal because the very compact two- and four-electron systems are overrepresented in the test set. Note that for the neon atom, which has a chemically more relevant electronic structure, the lowest absolute errors can be obtained at $\sim 35 \%$ of exact exchange for the density and the gradient, at $\sim 75 \%$ for the Laplacian, and at $\sim 35 \%$ for the three descriptors. Similarly, for molecules, we have found the minimal mean absolute error at $\sim 35 \%$ of exact exchange for the density and gradient, at $\sim 65 \%$ for the Laplacian, and at $\sim 45 \%$ for the three descriptors (Figure 3b).

For the atoms, the mean absolute DDXC in the PBE exchange-correlation energy is the lowest at high ratios of exact exchange $(90 \%)$, although this error is the most systematic at $25 \%$ of exact exchange mixing in the PBEh form (Figure 4a). The preference towards the full exact exchange originates again from the overrepresented very compact systems in the test set. For the neon atom, the mean absolute DDXC in the PBE exchange-correlation energy is the lowest at $\sim 40 \%$ of exact exchange mixing. For the molecules, the minimal density-driven exchange-correlation energy error appears at $\sim 40 \%$ as well, where the errors are also quite systematic (Figure 4b). At this ratio of exact exchange, the density-driven error in the PBE exchange-correlation energy is significantly lower on the PBEh densities than on the HF densities. Considering both the overall (three-descriptor) density error and the density-driven error in the PBE (or other semi-local) exchange-correlation energy, a fair compromise might be made around $40 \%$ of exact exchange mixing in the PBEh form for the chemically relevant densities $(e . g$., the neon atom or the molecules considered here). Notice that this is close to the global hybrid PBE38 method. ${ }^{51}$

Also seeking for an optimal PT2 correlation mixing in the PBE double hybrid form, we varied the ratio of PT2 correlation (from $0 \%$ to $100 \%$ by $5 \%$ ) at several ratios of exact exchange. Regarding the densities of the very compact atomic systems, the MP2 method seems to be the global minimum in the two-dimensional parameter space (Figure 5a). Interestingly, the PT2 correlation mixing corresponding to the one-dimensional minimum for a fixed $80 \%$ of exact exchange shifts to $\sim 55 \%$ for the molecular densities (Figure 5b). The global optimum seems to be around $95 \%$ of exact exchange and $80 \%$ of PT2 correlation.

For the very compressed atomic systems, the lowest density-driven error in the PBE exchangecorrelation energy seems to be around the MP2 method again (Figure 6a). For a fixed $80 \%$ of exact exchange, a minimum appears at 55\% of PT2 correlation mixing in the one-dimensional parameter space. For the molecules, the global minimum of the density-driven exchange-correlation energy error seems to be at $90 \%$ of exact exchange and $70 \%$ of PT2 correlation (Figure 6b). For a fixed $80 \%$ of exact exchange mixing, there is a minimum in the one-dimensional parameter space at $\sim 55 \%$ of PT2 correlation. The PT2 correlation ratio corresponding to the minimum for a fixed exact exchange ratio increases nearly linearly with the ratio of the exact exchange. Considering both the overall (threedescriptor) density errors and the density-driven errors in the PBE (or other semi-local) exchangecorrelation energy, the global optimum seems to be at $90-95 \%$ of exact exchange and $70-80 \%$ of PT2 correlation. Furthermore, there is a one-dimensional minimum for a fixed $80 \%$ of exact exchange at $\sim 55 \%$ of PT2 correlation for the examined molecules (with not too compact densities). Notice that the PBE-DH method with these mixing factors is not so far from the PBE0-2 method. 


\section{Conclusions}

According to the core theorem of density functional theory, the electron density, similar to the many-body wave function, contains all the information about the examined system. This property enables the error in the computed electron density to measure the proximity of a given density functional approximation to the exact functional. In the development of density functional approximations, it was assumed that the electron density improves together with the energetics. Recently, Medvedev et al. ${ }^{34}$ have argued that some modern density functional approximations with many empirical parameters and a very flexible functional form started producing worse electron densities despite their improved accuracy on energetics.

In this paper, we have assessed the performance of several density functional methods from various rungs of the Jacob's ladder of the density functional approximations. Our analysis uses a different methodology for the calculation of the density errors, in which the quantity measuring the accuracy of various methods is based on their average performance and is not size-extensive. Moreover, it can directly be applied to the comparison of non-centrosymmetric molecular densities. We have also constructed a small molecular test set. Notice that in the atomic test set of Medvedev et al., ${ }^{34}$ the chemically less relevant very compact atomic electron densities are overrepresented. Although their main message still holds, using an intensive error measure instead of an extensive one, aggregating the errors for different systems and descriptors by averaging instead of taking the maximum values, and applying a more balanced molecular test set lead to somewhat different conclusions about the performance of certain density functional approximations. For example, the meta-GGA level TPSS functional produces clearly better densities than the global hybrid PBE0 functional, and the empirically constructed M06L functional gives clearly better densities than the LDA method. We have also shown that better densities can be obtained with particular global double hybrid methods. For instance, the PBE0-2 double hybrid approaches the accuracy of the MP2 method for atomic densities and outperforms it for molecular ones.

In this work, we have also compared the density functional approximations with respect to the density-driven error in the semi-local exchange-correlation energy. According to our results, the densitydriven exchange-correlation energy errors do not always follow the same order that has been previously observed for the density errors. This is partially because the semi-local exchange-correlation energy functional we have used to indicate the density-driven exchange-correlation energy errors does not depend explicitly on the density Laplacian. Furthermore, the density-driven error in the semi-local exchange-correlation energy can occasionally integrate to almost zero. The density-driven exchangecorrelation energy errors are smaller on meta-GGA densities than on GGA densities. The quality of the parent semi-local functional also greatly influences the density-driven exchange-correlation energy errors on the global hybrid or double hybrid densities. The density-driven errors in the semi-local exchange-correlation energies are much smaller on the densities calculated with the best semi-local or non-local density functionals than on the HF densities. This tendency significantly intensifies on molecular densities.

Considering both the density errors and the density-driven errors in the semi-local exchangecorrelation energies, the best performers among their kind in the order of increasing accuracy are the PBE, the TPSS or SCAN, the TPSSh, and the PBE0-2 methods, all with non-empirical origin. In the PBE global hybrid form, a fair compromise can be made at $\sim 40 \%$ of exact exchange mixing, which is close to the PBE38 method. In the studied range, the global optimum in the PBE global double hybrid form appears at $90-95 \%$ of exact exchange and $70-80 \%$ of PT2 correlation, even though a one-dimensional minimum has been found for a fixed $80 \%$ of exact exchange at $55 \%$ of PT2 correlation mixing, which is not so far from the successful PBE0-2 global double hybrid method. Finally, we believe that our methodology can help developing more reliable hybrid or double hybrid functionals that approach more 
the exact functional than the ones fitted to chemical energetics.

\section{Additional information}

We make the computed molecular reference densities available at https://drive.google.com/open?id=0B4I8Qk8TWhVvS0xYVFN3OGxIaEU.

\section{Acknowledgments}

The authors thank the Hungarian National Higher Education and Research Network (NIIF) for the computer time.

\section{Supporting information}

The computed atomic densities, further information about the density errors and density-driven exchange-correlation energy errors, as well as detailed results for the discussed methods. This information is available free of charge via the Internet at http://pubs.acs.org/. 


\section{Figures}

a)

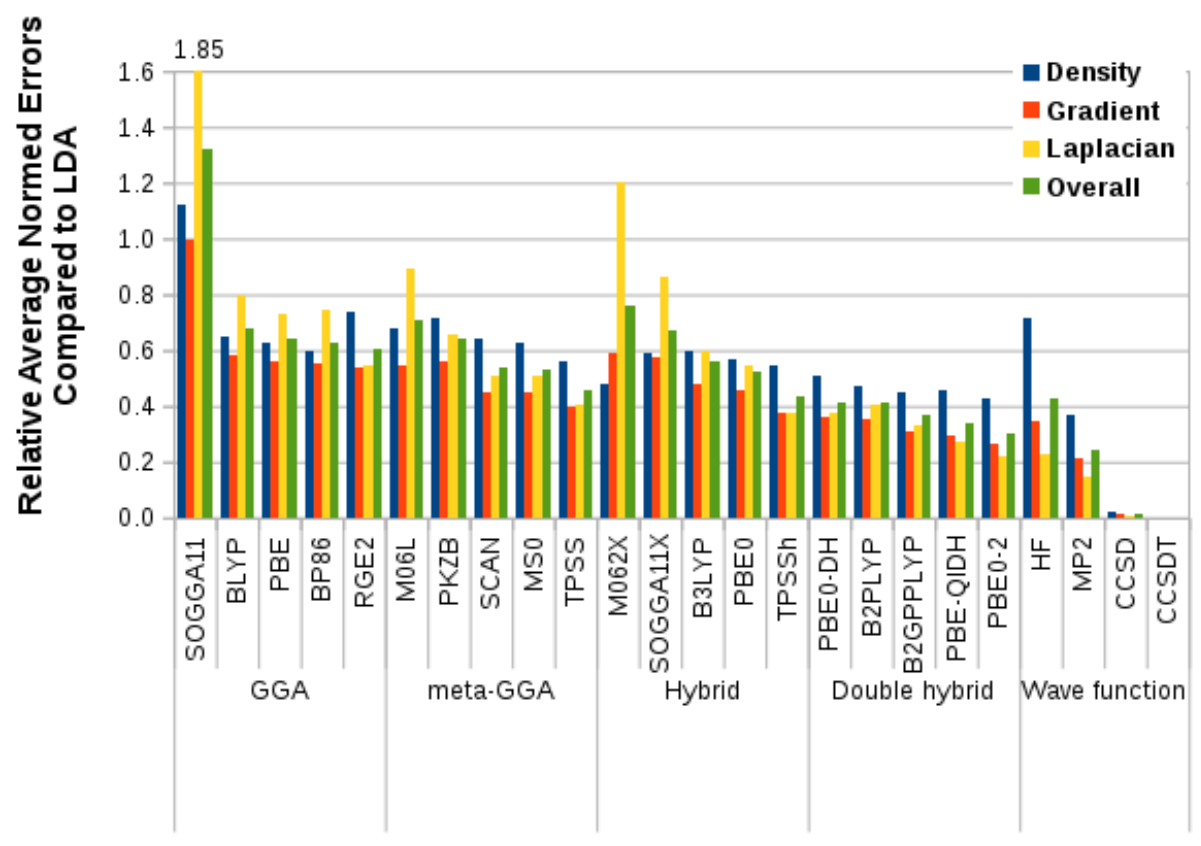

Method Applied in the Self-Consistent Density Calculation

b)

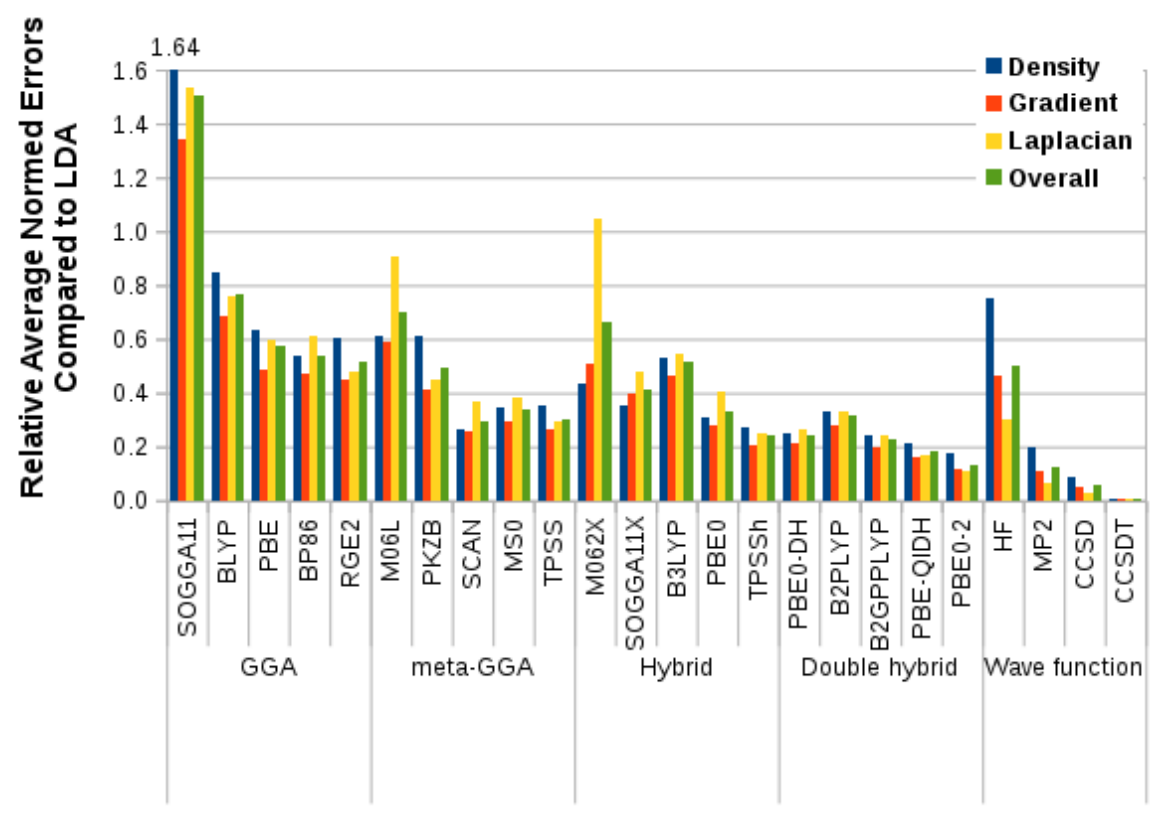

Method Applied in the Self-Consistent Density Calculation

Figure 1 Relative average normed errors (RANE) for the atoms (a) and molecules (b) calculated with various methods compared to the local density approximation (LDA) 
a)

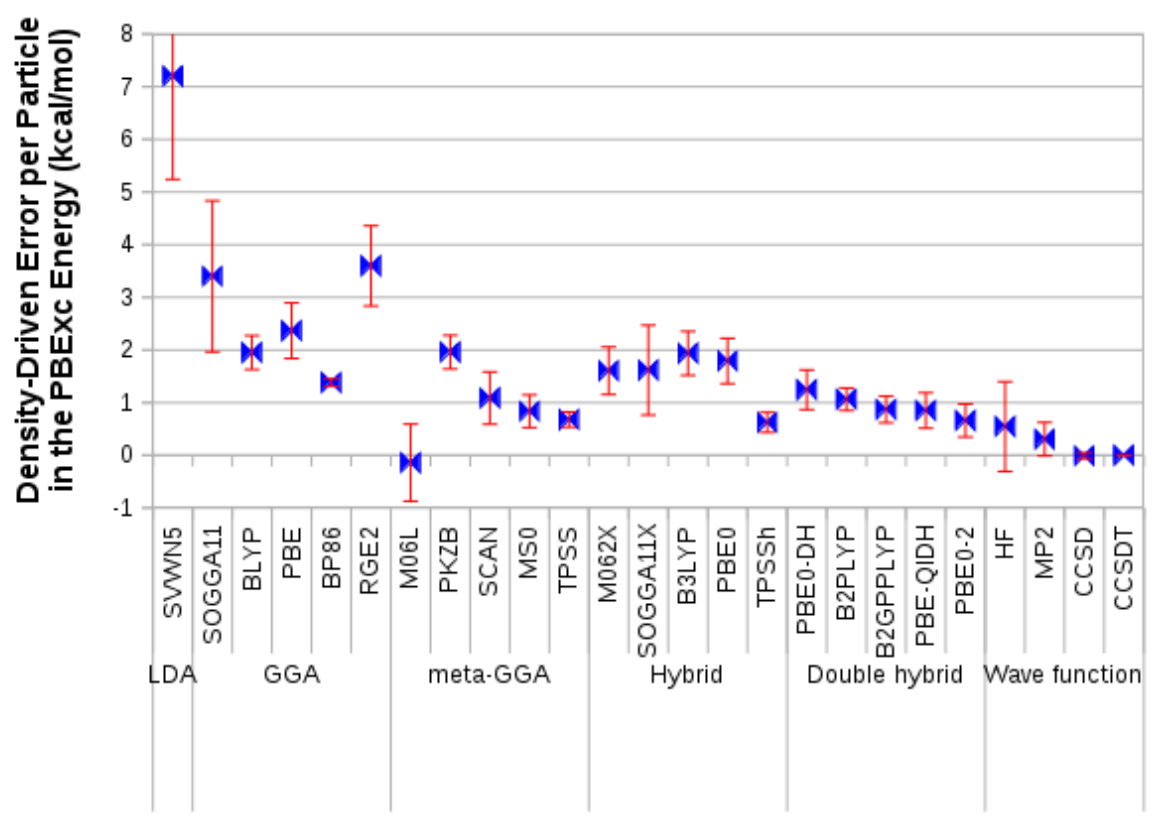

Method Applied in the Self-Consistent Density Calculation

b)

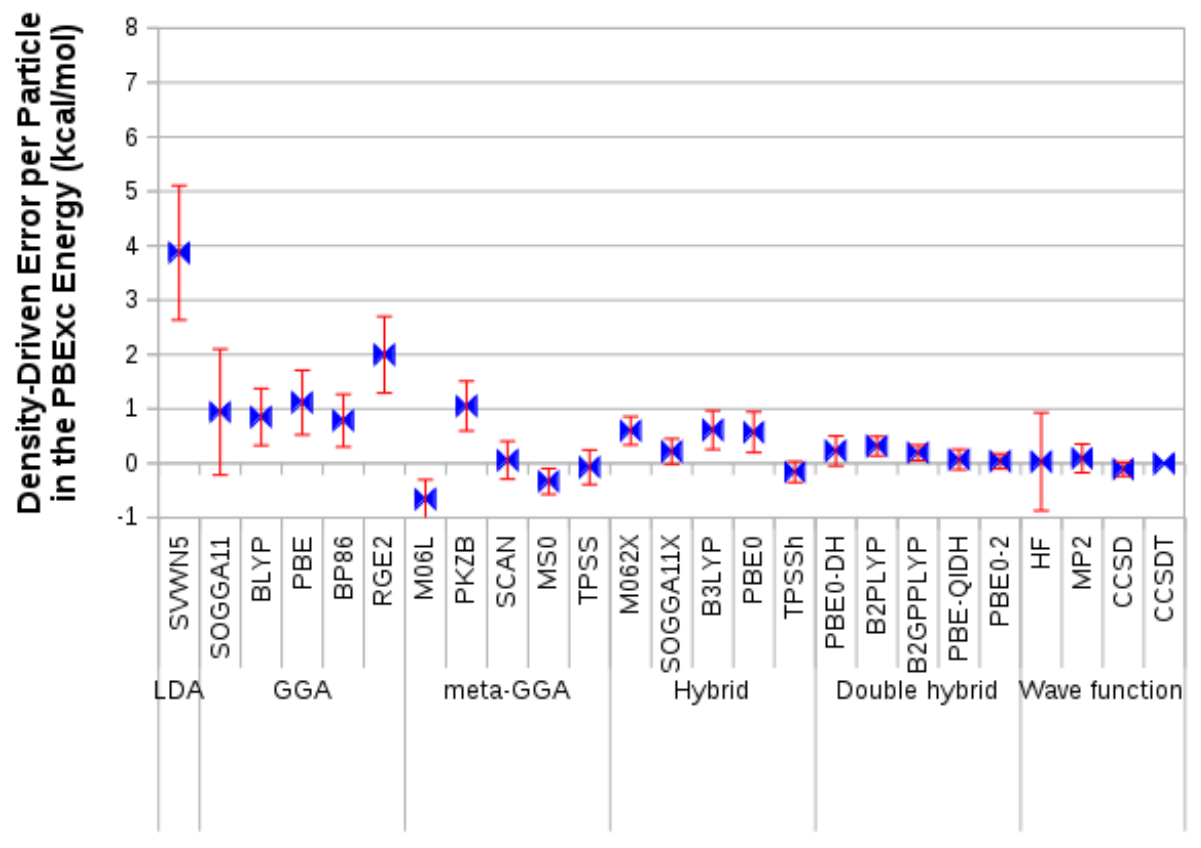

Method Applied in the Self-Consistent Density Calculation

Figure 2 Density-driven error per particle in the PBE exchange-correlation energy (DDXC) evaluated on densities calculated with various methods for the atomic (a) and molecular (b) test sets. (The error bars show the standard deviation for the errors.) 
a)

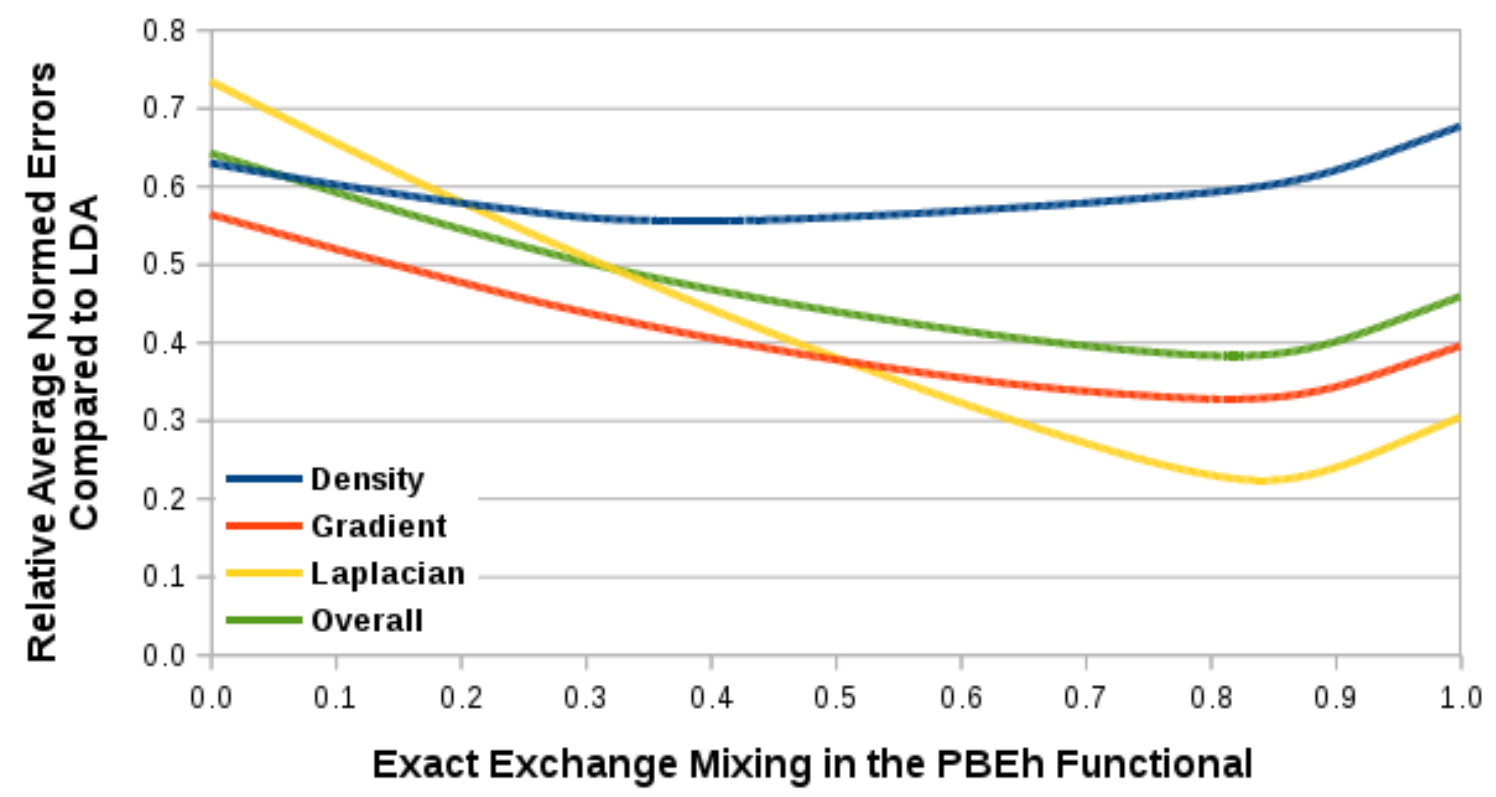

b)

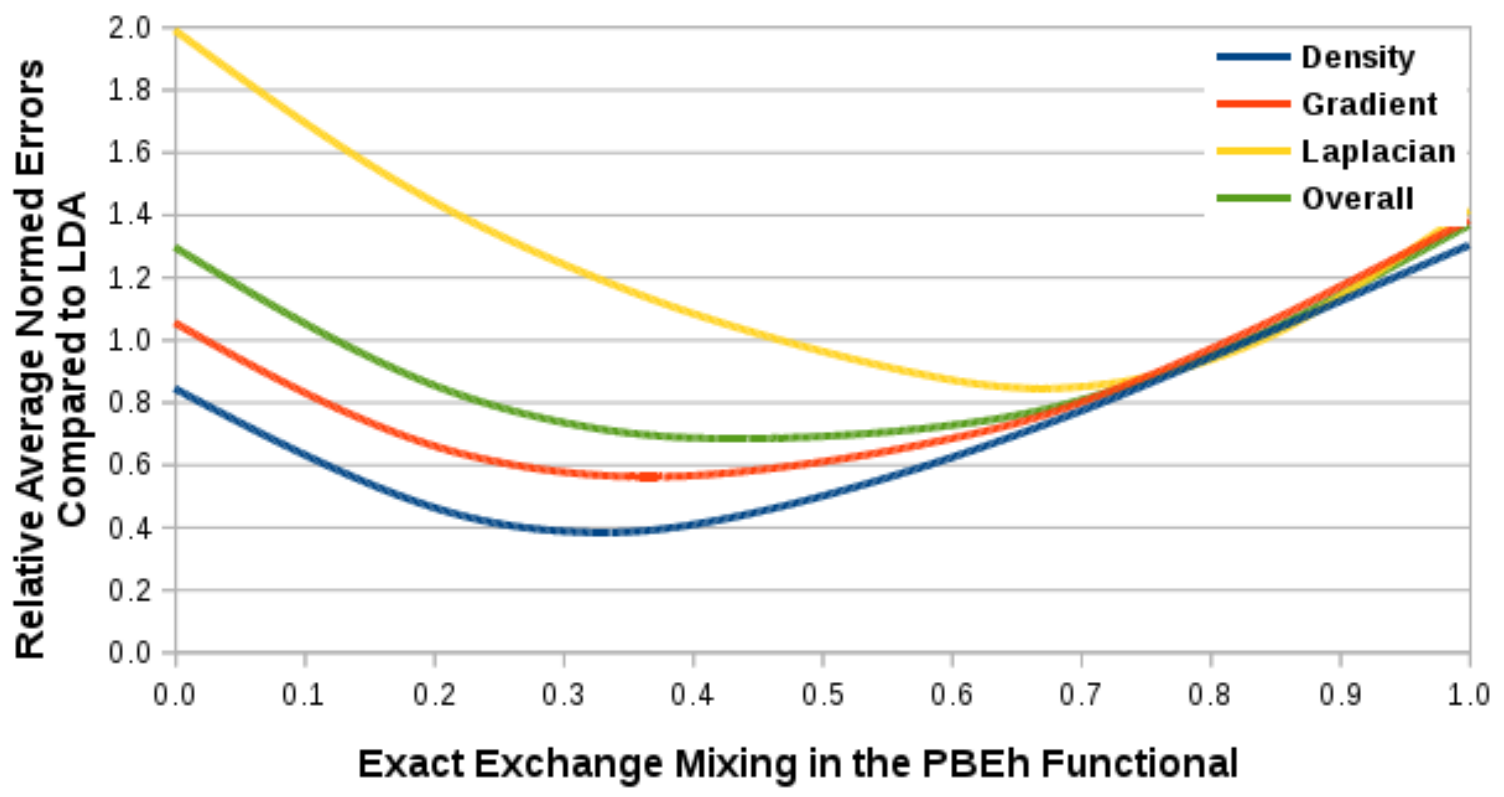

Figure 3 Dependence of the relative average normed errors (RANE) for the atoms (a) and molecules (b) on the exact exchange mixing in the PBEh functional compared to the local density approximation (LDA) 
a)

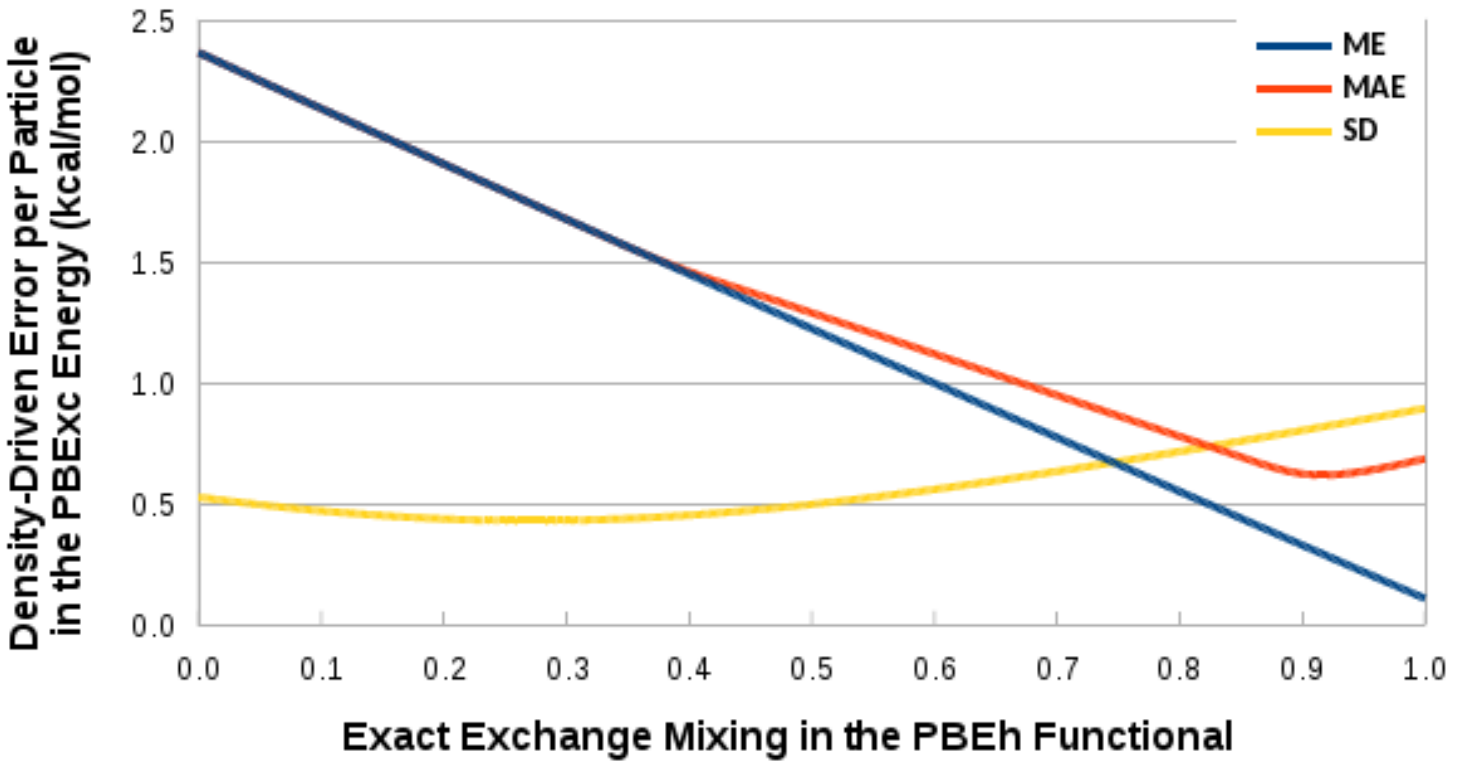

b)

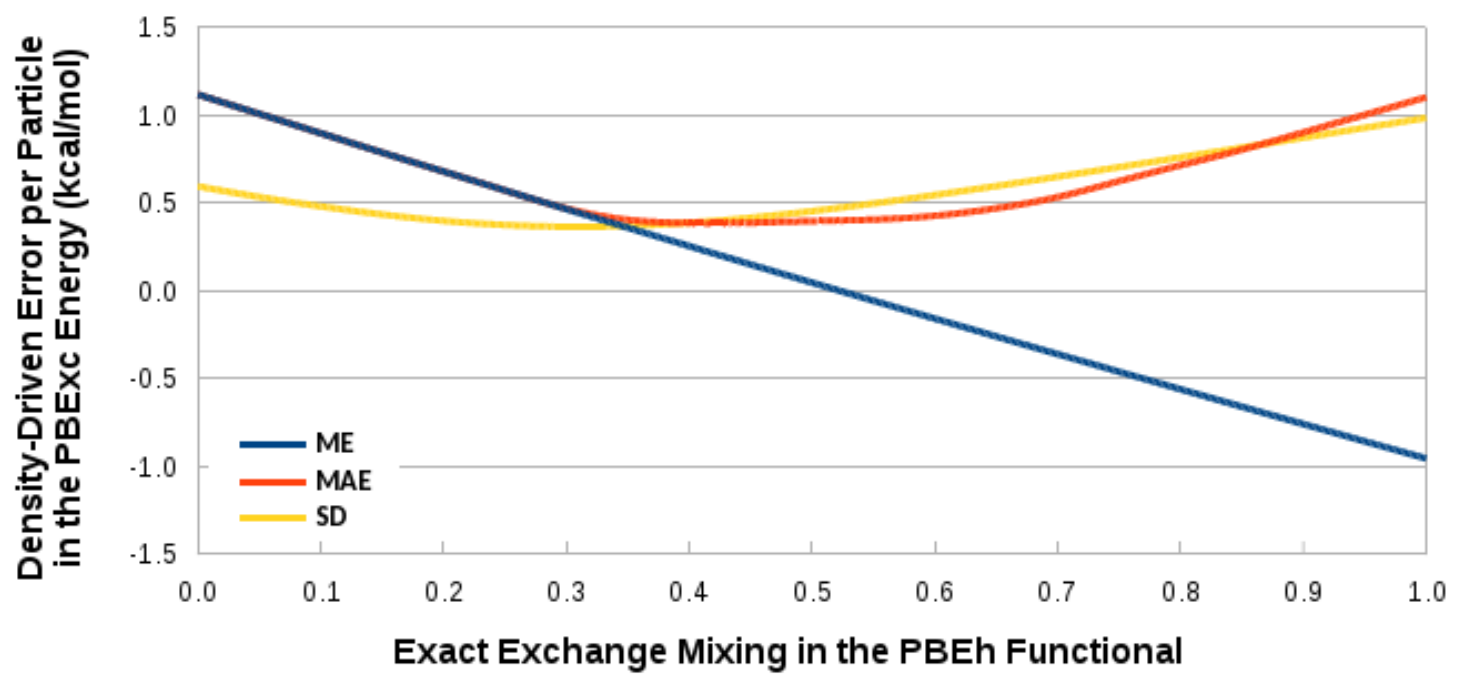

Figure 4 Dependence of the density-driven error per particle in the PBE exchange-correlation energy (DDXC) of the atoms (a) and molecules (b) on the exact exchange mixing in the PBEh densities (ME: mean error; MAE: mean absolute error; SD: standard deviation of the errors) 
a)

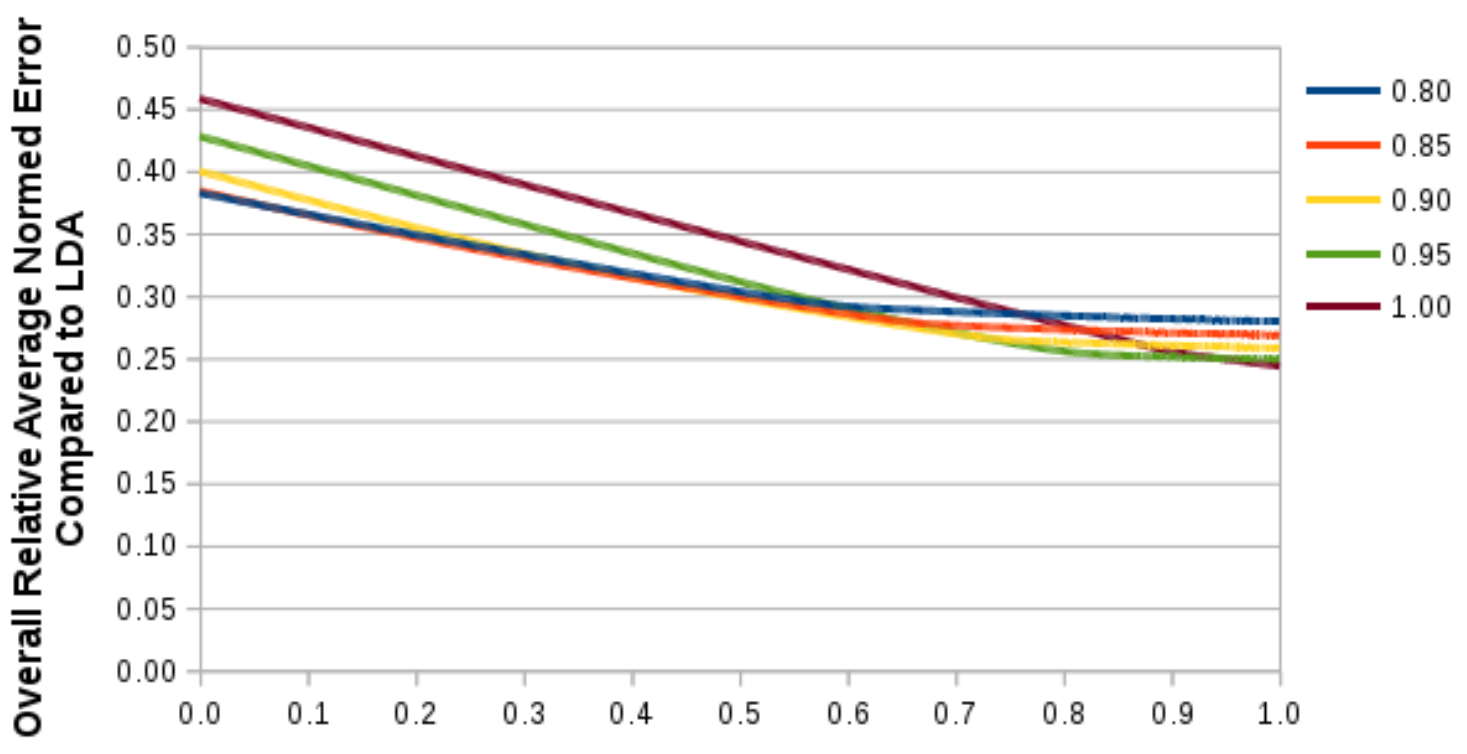

PT2 Correlation Mixing in the PBE-DH Functional

b)

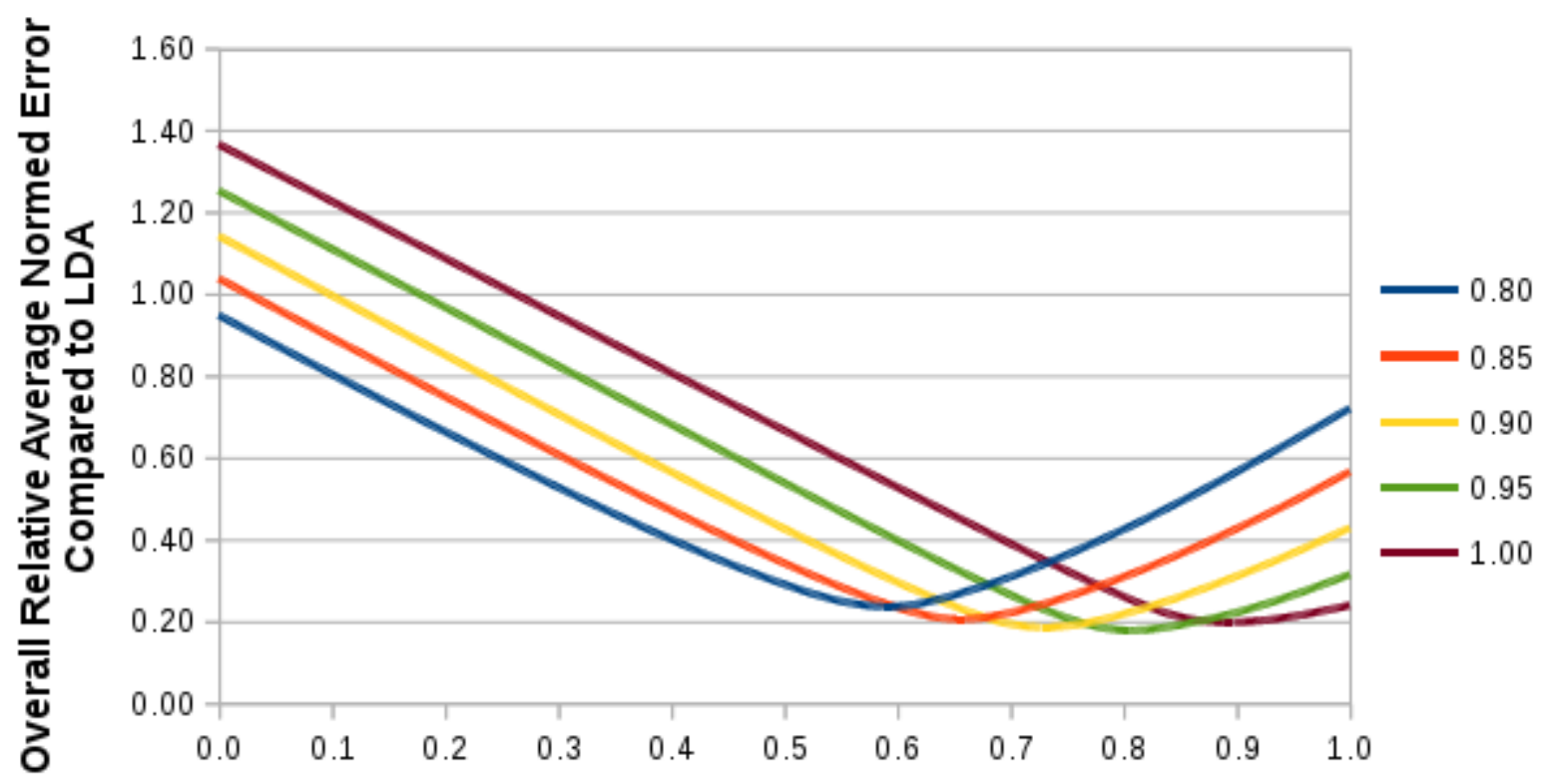

PT2 Correlation Mixing in the PBE-DH Functional

Figure 5 Dependence of the overall (three-descriptor) relative average normed error (RANE) on the second-order perturbative correlation mixing in the PBE-DH functional relative to the local density approximation for various exact exchange mixing ratios (displayed in the key) for the atomic (a) and molecular (b) test sets 
a)

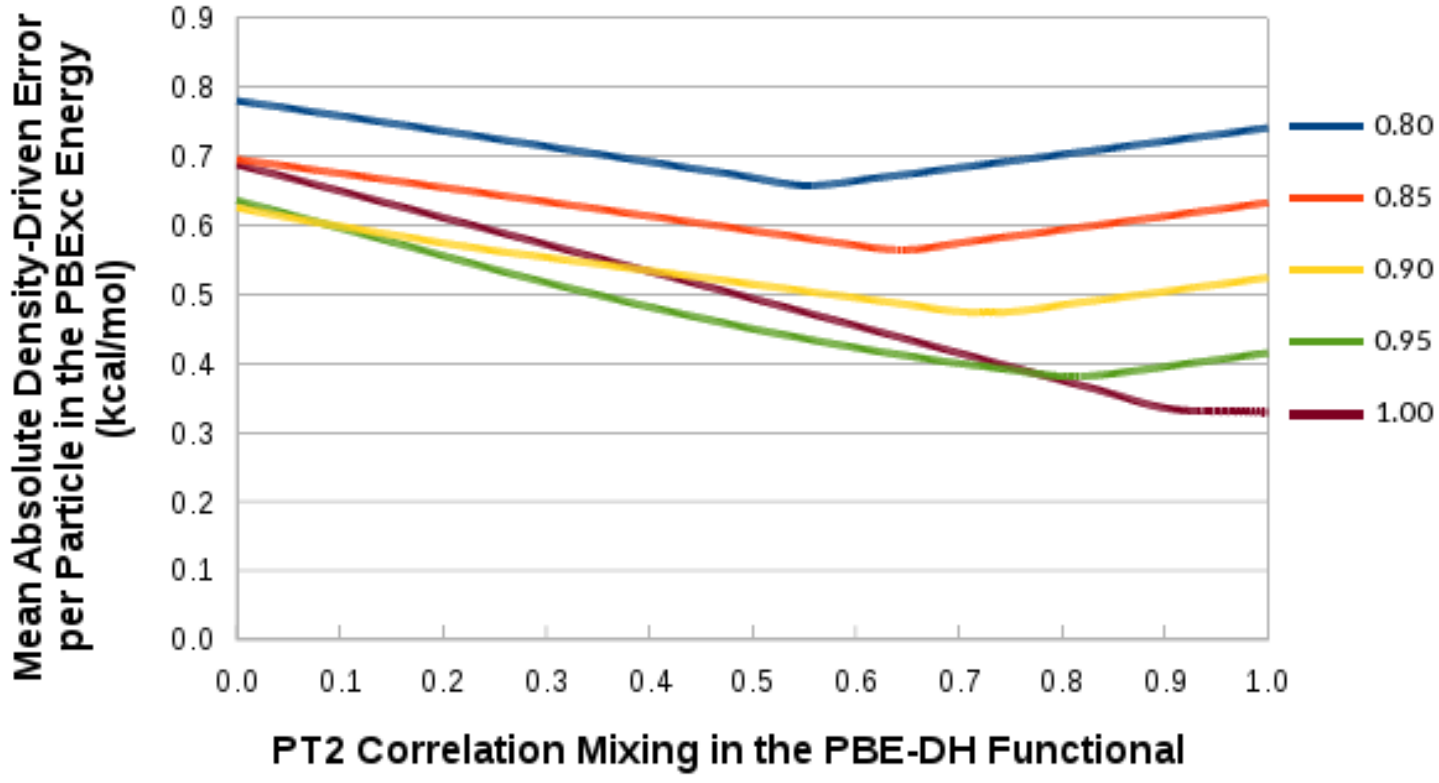

b)

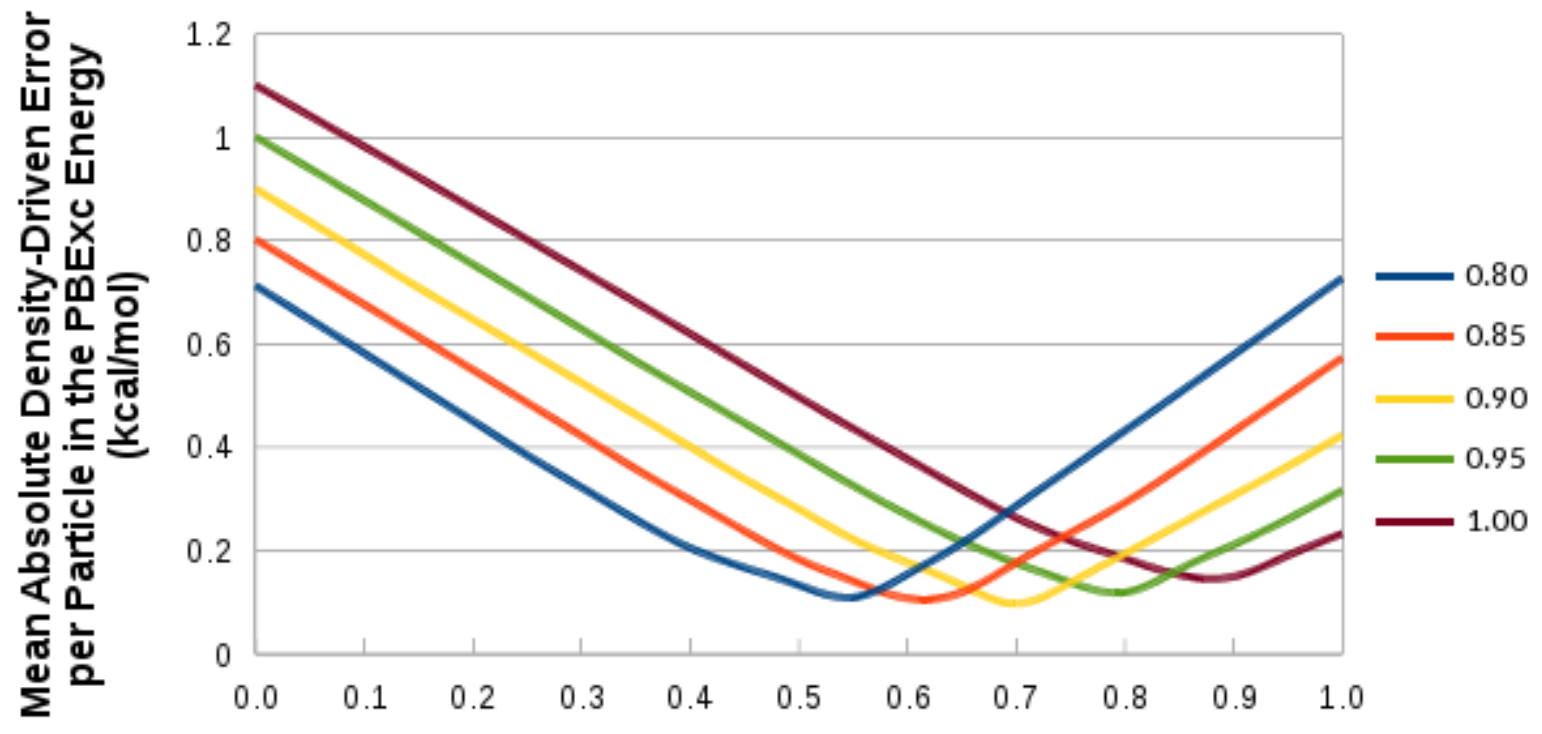

PT2 Correlation Mixing in the PBE-DH Functional

Figure 6 Dependence of the density-driven error per particle in the PBE exchange-correlation energy (DDXC) of the atoms (a) and molecules (b) on the second-order perturbative correlation mixing in the $\mathrm{PBE}-\mathrm{DH}$ densities for various exact exchange mixing ratios (displayed in the key) 


\section{References}

1 Parr, R. G.; Yang, W. Density Functional Theory of Atoms and Molecules; Springer: Oxford/New York, 1989.

2 Becke, A. D. Perspective: Fifty years of density-functional theory in chemical physics. J. Chem. Phys. 2014, 140, $18 \mathrm{~A} 301$.

3 Hohenberg, P.; Kohn, W. Inhomogeneous electron gas. Phys. Rev. 1964, 136, B864.

4 Kohn, W.; Sham, L. J. Self-consistent equations including exchange and correlation effects. Phys. Rev. 1965, 140, A1133.

5 Perdew, J. P.; Ruzsinszky, A.; Tao, J.; Staroverov, V. N.; Scuseria, G. E.; Csonka, G. I. Prescription for the design and selection of density functional approximations: More constraint satisfaction with fewer fits. J. Chem. Phys. 2005, 123, 062201.

6 Csonka, G. I.; Vydrov, O. A.; Scuseria, G. E.; Ruzsinszky, A.; Perdew, J. P. Diminished gradient dependence of density functionals: Constraint satisfaction and self-interaction correction. J. Chem. Phys. 2007, 126, 244107.

7 Perdew, J. P.; Staroverov, V. N.; Tao, J.; Scuseria, G. E. Density functional with full exact exchange, balanced nonlocality of correlation, and constraint satisfaction. Phys. Rev. A 2008, 78, 052513.

8 Zhao, Y.; Schultz, N. E.; Truhlar, D. G. Design of Density Functionals by Combining the Method of Constraint Satisfaction with Parametrization for Thermochemistry, Thermochemical Kinetics, and Noncovalent Interactions. J. Chem. Theory Comput. 2006, 2, 364-382.

9 Zhao, Y.; Truhlar, D. G. Density functionals with broad applicability in chemistry. Acc. Chem. Res. 2008, 41, 157-167. 10 Yu, H. S.; Li, S. L.; Truhlar, D. G. Perspective: Kohn-Sham density functional theory descending a staircase. J. Chem. Phys. 2016, 145, 130901.

11 Xu, X.; Zhang, W.; Tang, M.; Truhlar, D. G. Do Practical Standard Coupled Cluster Calculations Agree Better than Kohn-Sham Calculations with Currently Available Functionals When Compared to the Best Available Experimental Data for Dissociation Energies of Bonds to 3d Transition Metals? J. Chem. Theory Comput. 2015, 11, 2036-2052.

12 Perdew, J. P.; Sun, J.; Ruzsinszky, A.; Mezei, P. D.; Csonka, G. I. Why Density Functionals Should Not Be Judged Primarily by Atomization Energies. Period. Polytech., Chem. Eng. 2016, 60, 2.

13 Mardirossian, N.; Head-Gordon, M. How Accurate Are the Minnesota Density Functionals for Noncovalent Interactions, Isomerization Energies, Thermochemistry, and Barrier Heights Involving Molecules Composed of MainGroup Elements? J. Chem. Theory Comput. 2016, 12, 4303-4325.

14 Becke, A. D. Density-functional thermochemistry. III. The role of exact exchange. J. Chem. Phys. 1993, 98, 56485652.

15 Boyd, R. J.; Wang, J.; Eriksson, L. A. The electron density as calculated from density functional theory. Recent Adv. Density Funct. Methods 1995, 1, 369-401.

16 Csonka, G. I.; Nguyen, N. A.; Kolossváry, I. Simple tests for density functional methods. J. Comput. Chem. 1997, 18, 1534-1545.

17 Becke, A. D. Density-functional exchange-energy approximation with correct asymptotic behavior. Phys. Rev. A 1988, 38, 3098-3100.

18 Perdew, J. P. Density-functional approximation for the correlation energy of the inhomogeneous electron gas. Phys.

Rev. B 1986, 33, 8822-8824.

19 van Leeuwen, R.; Baerends, E. J. An analysis of nonlocal density functionals in chemical bonding. Int. J. Quantum Chem. 1994, 52, 711-730.

20 Bochevarov, A. D.; Friesner, R. A. The densities produced by the density functional theory: Comparison to full configuration interaction. J. Chem. Phys. 2008, 128, 034102.

21 Handy, N. C.; Cohen, A. J. Left-right correlation energy. Mol. Phys. 2001, 99, 403-412.

22 Vosko, S. H.; Wilk, L.; Nusair, M. Accurate spin-dependent electron liquid correlation energies for local spin density calculations: a critical analysis. Can J. Phys. 1980, 58, 1200-1211.

23 Lee, C.; Yang, W.; Parr, R. G. Development of the Colle-Salvetti correlation-energy formula into a functional of the electron density. Phys. Rev. B 1988, 37, 785-789.

24 Grabowski, I.; Teale, A. M.; Śmiga, S.; Bartlett, R. J. Comparing ab initio density-functional and wave function theories: The impact of correlation on the electronic density and the role of the correlation potential. J. Chem. Phys. 2011, $135,114111$.

25 Grabowski, I.; Fabiano, E.; Teale, A. M.; Śmiga, S.; Buksztel, A.; Della Sala, F. Orbital-dependent second-order scaled-opposite-spin correlation functionals in the optimized effective potential method. J. Chem. Phys. 2014, 141, 024113. 26 Tognetti, V.; Joubert, L. On the influence of density functional approximations on some local Bader's atoms-inmolecules properties. J. Phys. Chem. A 2011, 115, 5505-5515.

27 Tao, J.; Perdew, J. P.; Staroverov, V. N.; Scuseria, G. E. Climbing the density functional ladder: Nonempirical meta- 
generalized gradient approximation designed for molecules and solids. Phys. Rev. Lett. 2003, $91,146401$.

28 Perdew, J. P.; Burke, K.; Ernzerhof, M. Generalized gradient approximation made simple. Phys. Rev. Lett. 1996, 77, 3865-3868.

29 Hamprecht, F. A.; Cohen, A. J.; Tozer, D. J.; Handy, N. C. Development and assessment of new exchange-correlation functionals. J. Chem. Phys. 1998, 109, 6264-6271.

30 Boese, A. D.; Doltsinis, N. L.; Handy, N. C.; Sprik, M. New generalized gradient approximation functionals. J. Chem. Phys. 2000, 112, 1670-1678.

31 Boese, A. D.; Handy, N. C. A new parametrization of exchange-correlation generalized gradient approximation functionals. J. Chem. Phys. 2001, 114, 5497-5503.

32 Cruz, F. G.; Lam, K-C.; Burke, K. Exchange-Correlation Energy Density from Virial Theorem. J. Phys. Chem. A 1998, 102, 4911-4917.

33 Kim, M-C.; Sim, E.; Burke, K. Understanding and reducing errors in density functional calculations. Phys. Rev. Lett. 2013, 111, 073003 .

34 Medvedev, M. G.; Bushimarinov, I. S.; Sun, J.; Perdew, J. P.; Lyssenko, K. A. Density functional theory is straying from the path toward the exact functional. Science 2017, 355, 49-52.

35 Graziano, G. Quantum chemistry: DFT's midlife crisis. Nat. Rev. Chem. 2017, 1, 0019.

36 Hammes-Schiffer, S. A conundrum for density functional theory. Science 2017, 355, 28-29.

37 Kepp, K. P. Comment on "Density functional theory is straying from the path toward the exact functional". Science 2017, 356, 496b.

38 Korth, M. Density Functional Theory: Not Quite the Right Answer for the Right Reason Yet. Angew. Chem. Int. Ed. 2017, 56, 2-5.

39 Adamo, C.; Barone, V. Toward reliable density functional methods without adjustable parameters: The PBE0 model. J. Chem. Phys. 1999, 110, 6158-6170.

40 Medvedev, M. G.; Bushimarinov, I. S.; Sun, J.; Perdew, J. P.; Lyssenko, K. A. Response to Comment on "Density functional theory is straying from the path toward the exact functional". Science 2017, 355, 496c.

41 Brorsen, K. R.; Yang, Y.; Pak, M. V.; Hammes-Schiffer, S. Is the Accuracy of Density Functional Theory for Atomization Energies and Densities in Bonding Regions Correlated? J. Phys. Chem. Lett. 2017, 8, $2076-2081$.

42 Gould, T. What Makes a Density Functional Approximation Good? Insights from the Left Fukui Function. J. Chem. Theory Comput. 2017, 13, 2373-2377.

43 Peverati, R.; Truhlar, D. G. A global hybrid generalized gradient approximation to the exchange-correlation functional that satisfies the second-order density-gradient constraint and has broad applicability in chemistry. J. Chem. Phys. 2011, $135,191102$.

44 Staroverov, V. N.; Scuseria, G. E. Comparative assessment of a new nonempirical density functional: Molecules and hydrogen-bonded complexes. J. Chem. Phys. 2003, 119, 12129-12137.

45 Grimme, S. Semiempirical hybrid density functional with perturbative second-order correlation. J. Chem. Phys. 2006, $124,034108$.

46 Verma, P.; Truhlar, D. G. Can Kohn-Sham density functional theory predict accurate charge distributions for both single-reference and multi-reference molecules? Phys. Chem. Chem. Phys. 2017, 19, 12898-12912.

47 Zhao, Y.; Truhlar, D. G. Design of Density Functionals That Are Broadly Accurate for Thermochemistry,

Thermochemical Kinetics, and Nonbonded Interactions. J. Phys. Chem. A 2005, 109, 5656-5667.

48 Paier, J.; Marsman, M.; Hummer, K.; Kresse, G.; Gerber, I. C.; Ángyán, J. G. Screened hybrid density functionals applied to solids. J. Chem. Phys. 2006, 124, 154709.

49 Perdew, J. P.; Schmidt, K. Jacob's ladder of density functional approximations for the exchange-correlation energy.

AIP Conf. Proc. 2001, 577, 1-20.

50 Csonka, G. I.; Perdew, J. P.; Ruzsinszky, A. Global hybrid functionals: a look at the engine under the hood. J. Chem. Theory Comput. 2010, 6, 3688-3703.

51 Grimme, S.; Antony, J.; Ehrlich, S.; Krieg, H. A consistent and accurate ab initio parametrization of density functional dispersion correction (DFT-D) for the 94 elements H-Pu. J. Chem. Phys. 2010, 132, 154104.

52 Brémond, E.; Adamo, C. Seeking for parameter-free double-hybrid functionals: the PBE0-DH model. J. Chem. Phys. 2011, 135, 024106.

53 Brémond, E.; Sancho-García, J. C.; Pérez-Jimenez, Á. J.; Adamo, C. Communication: Double-hybrid functionals from adiabatic-connection: The QIDH model. J. Chem. Phys. 2014, 141, 031101.

54 Chai, J-D.; Mao, S-P. Seeking for reliable double-hybrid density functionals without fitting parameters: the PBE0-2 functional. Chem. Phys. Lett. 2012, 538, 121-125.

55 Mrcc, a quantum chemical program suite written by M. Kállay, Z. Rolik, J. Csontos, P. Nagy, G. Samu, D. Mester, I. Ladjánszki, L. Szegedy, B. Ladóczki, K. Petrov, M. Farkas, and B. Hégely. See also www.mrcc.hu. 
56 Mura, M. E.; Knowles, P. J. Improved radial grids for quadrature in molecular density-functional calculations. $J$. Chem. Phys. 1996, 104, 9848-9858.

57 Lebedev, V. I.; Laikov, D. N. A quadrature formula for the sphere of the 131st algebraic order of accuracy. Dokl. Math. 1999, 59, 477-481.

58 Dunning, T. H. Gaussian basis sets for use in correlated molecular calculations. I. The atoms boron through neon and hydrogen. J. Chem. Phys. 1989, 90, 1007.

59 Peterson, K. A.; Dunning Jr., T. H. Accurate correlation consistent basis sets for molecular core-valence correlation effects: The second row atoms Al-Ar, and the first row atoms B-Ne revisited. J. Chem. Phys. 2002, 117, 10548.

60 NIST Computational Chemistry Comparison and Benchmark Database, NIST Standard Reference Database Number 101, Release 18, October 2016, Johnson, R. D., III, Ed. http://cccbdb.nist.gov/ (accessed March 30, 2017)

61 Peverati, R.; Zhao, Y.; Truhlar, D. G. Generalized gradient approximation that recovers the second-order densitygradient expansion with optimized across-the-board performance. J. Phys. Chem. Lett. 2011, 2, 1991-1997.

62 Ruzsinszky, A.; Csonka, G. I.; Scuseria, G. E. Regularized gradient expansion for atoms, molecules, and solids. J. Chem. Theory Comput. 2009, 5, 763-769.

63 Perdew, J. P.; Kurth, S.; Zupan, A.; Blaha, P. Accurate density functional with correct formal properties: A step beyond the generalized gradient approximation. Phys. Rev. Lett. 1999, 82, 2544-2547.

64 Zhao, Y.; Truhlar, D. G. A new local density functional for main-group thermochemistry, transition metal bonding, thermochemical kinetics, and noncovalent interactions. J. Chem. Phys. 2006, 125, 194101.

65 Sun, J.; Ruzsinszky, A.; Perdew, J. P. Strongly constrained and appropriately normed semilocal density functional. Phys. Rev. Lett. 2015, 115, 036402.

66 Sun, J.; Xiao, B.; Ruzsinszky, A. Communication: Effect of the orbital-overlap dependence in the meta generalized gradient approximation. J. Chem. Phys. 2012, 137, 051101.

67 Zhao, Y.; Truhlar, D. G. The M06 suite of density functionals for main group thermochemistry, thermochemical kinetics, noncovalent interactions, excited states, and transition elements: two new functionals and systematic testing of four M06-class functionals and 12 other functionals. Theor. Chem. Acc. 2008, 120, 215-241.

68 Karton, A.; Tarnopolsky, A.; Lamère, J-F.; Schatz, G. C.; Martin, J. M. L. Highly Accurate First-Principles Benchmark Data Sets for the Parametrization and Validation of Density Functional and Other Approximate Methods. Derivation of a Robust, Generally Applicable, Double-Hybrid Functional for Thermochemistry and Thermochemical Kinetics. J. Phys. Chem. A 2008, 112, 12868-12886.

69 Slater, J. C. A simplification of the Hartree-Fock method. Phys. Rev. 1951, 81, 385-390. 


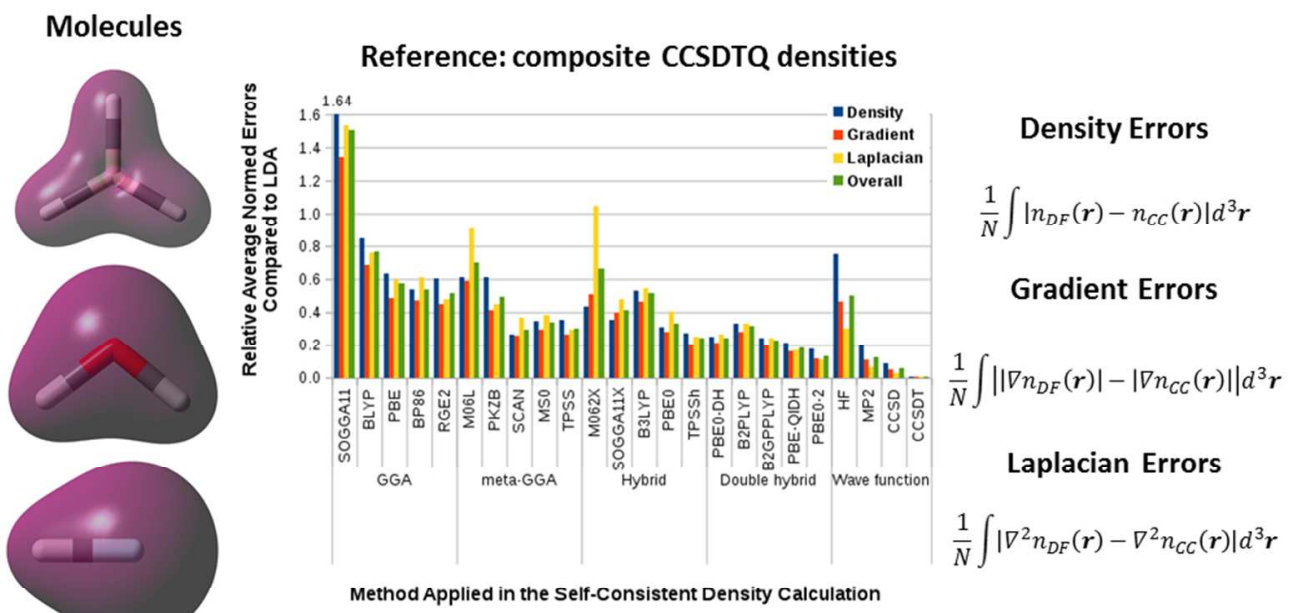

TOC graphic

$85 \times 47 \mathrm{~mm}(300 \times 300$ DPI $)$ 